\title{
Association of the Irregular 3-Dimensional Shape of White Matter Hyperintensities with Cognitive Function
}

\author{
Ji Won Han ${ }^{a, b}$ Hyunna Lee ${ }^{c}$ Subin Lee ${ }^{d}$ Haejin Kim $^{e}$ Grace Eun Kim $^{d}$ \\ Jong Bin Bae ${ }^{a}$ Seung Wan Suh ${ }^{f}$ Jae Hyoung Kim ${ }^{g}$ Ki Woong Kim ${ }^{\text {a, b, d }}$ \\ aDepartment of Neuropsychiatry, Seoul National University Bundang Hospital, Seongnam, South Korea; \\ bepartment of Psychiatry, College of Medicine, Seoul National University, Seoul, South Korea; 'Health Innovation \\ Big Data Center, Asan Institute for Life Science, Asan Medical Center, University of Ulsan College of Medicine, \\ Seoul, South Korea; 'Department of Brain \& Cognitive Sciences, Seoul National University, Seoul, South Korea; \\ eDepartment of Basic Science, Hongik University, Seoul, Sejong, South Korea; fDepartment of Psychiatry, Kangdong \\ Sacred Heart Hospital, Hallym University College of Medicine, Seoul, South Korea; 9Department of Radiology, Seoul \\ National University Bundang Hospital, Seongnam, South Korea
}

\section{Keywords}

White matter hyperintensities - Shape · Dementia - Ischemia . Cognition

\begin{abstract}
Introduction: The irregular shapes of white matter hyperintensities (WMHs) are associated with poor cognitive function, diabetes, or lacunes. However, the association between the WMH shape and dementia remains understudied. We investigated the association between the calculated shape index of WMH and the diagnosis of dementia and cognitive function. Methods: The inverse sphericity index (ISI $\left.\right|_{\mathrm{WMH}}$ ) and volume of WMHs ( $\left.\mathrm{VOL}_{\mathrm{WMH}}\right)$ were compared among 82 participants with normal cognition, 82 with Alzheimer's dementia (AD), and 82 with subcortical vascular dementia (SVD). We examined the associations of $I S I_{\mathrm{WMH}}$ and $\mathrm{VOL}_{\mathrm{WMH}}$ with the modified Hachinski Ischemic Score (mHIS), diagnosis of AD and SVD, and cognitive test scores, using linear, multinomial, or hierarchical linear regression models. Results: The mHIS was associated with both ISI $\mathrm{WMH}_{\mathrm{MH}}(\beta=0.326, p<0.001)$ and $\operatorname{VOL}_{W M H}(\beta=0.299, p<0.001)$. Both ISI $I_{W M H}$ and VOL ${ }_{W M H}$ were associated with the SVD diagnosis (odds ratio $[\mathrm{OR}]=2.685$,
\end{abstract} $A D$. The SVD diagnosis was better explained when the multinomial regression model included both $\mathrm{ISI}_{\mathrm{WMH}}$ and $\mathrm{VOL}_{\mathrm{WMH}}$ instead of $\mathrm{VOL}_{\mathrm{WMH}}$ alone $\left(\mathrm{X}^{2}=20.768, \mathrm{df}=2, p<0.001\right)$. The Trail Making Test-D (TMT-D) scores of the SVD patients were associated with both $I S I_{W M H}(\beta=0.308)$ and $\mathrm{VOL}_{W M H}(\beta=$ 0.293). Conclusion: An irregular WMH shape may be associated with the high cerebrovascular component of cognitive impairment and the diagnosis and low cognitive flexibility of SVD, which may improve the prediction of SVD diagnosis when used in combination with WMH volume.

(c) 2021 S. Karger AG, Basel

\section{Introduction}

It is challenging to determine the degree to which white matter hyperintensities (WMHs) account for observed cognitive deficits and/or dementia [1]. The total volume and location of WMHs are the features that are most investigated as its cognitive correlates. While some studies do not support the relationship between these features and cognition [2-5], recent studies have reported

Correspondence to:

Ki Woong Kim, kwkimmd@snu.ac.kr 
that the locations of WMHs are more relevant in explaining the variance in cognitive functioning than the global $\mathrm{WMH}$ volumes [6-10], particularly for strategic white matter tracts in $\mathrm{WMH}[6,8-10]$.

The shape features of WMHs have also been investigated for their association with cognition. Periventricular smooth or spherical WMHs are thought to have less impact on cognition than more irregular and confluent WMHs [11-13]. Moreover, irregular WMHs have more ischemic histopathological findings [11-17], which may be provoked by aging and vascular risk factors [18].

Recent studies have evaluated the shape features of WMHs by computing specific indexes, as visual evaluation may cause intra- and inter-rater variability [19$22]$. These studies have consistently reported that an irregular shape index is associated with poor cognitive function [19], diabetes [20], or lacunes [21]. Lange et al. [19] calculated the "confluency sum score (COSU)" as a marker of the total shape irregularity of WMHs and observed a more significant association between mental flexibility and COSU than mental flexibility and WMH volume in a population with mixed diagnoses. However, most previous studies on the computed shape indexes of WMHs have focused on either cognitive test scores [19] or cerebrovascular risk factors related to cognition $[20,21]$ and did not investigate their association with dementia.

In this study, we examined the associations of the volume and shape index of WMH with (1) the cerebrovascular component of cognitive impairment measured by the modified Hachinski Ischemic Score (mHIS) [23-26] and (2) the diagnosis and (3) cognitive performance of patients with Alzheimer's dementia (AD) and subcortical vascular dementia (SVD). We hypothesized that the shape of WMHs had a stronger association with the mHIS than the volume, and it may improve the prediction of SVD diagnosis when used in combination with WMH volume.

\section{Materials and Methods}

\section{Study Population}

We enrolled 246 participants (82 with normal cognition [NC], 82 with AD, and 82 with SVD). Of them, 52 were from the Korean Longitudinal Study on Cognitive Aging and Dementia [27] and 194 were visitors to the Dementia Clinic of Seoul National University Bundang Hospital. The inclusion criteria were as follows: (1) a WMH volume above $1,000 \mathrm{~mm}^{3}$, which is the threshold for producing the shape index, and (2) an absence of major psychiatric disorders (including depressive disorders, schizophrenia, and substance use disorders), major neurologic disorders (including movement disorders, multiple sclerosis, brain tumor, and normal pressure hydrocephalus), other types of dementia except $\mathrm{AD}$ and SVD, or severe medical conditions that may influence mood and cognition. We excluded participants with cortical and/or corticosubcortical non-lacunar territorial infarcts and watershed infarcts, or hemorrhages on their brain imaging.

\section{Assessments}

Geriatric neuropsychiatrists with expertise in dementia research administered a face-to-face standardized diagnostic interview, in addition to physical and neurological examinations, using the Korean version of the Consortium to Establish a Registry for Alzheimer's Disease Assessment Packet Clinical Assessment Battery (CERAD-K-C) [28], to diagnose cognitive disorders. They further administered the mHIS [23] to evaluate the cerebrovascular component of cognitive impairment [25]. The HIS has been used in previous studies to represent vascular risk factors $[26,29]$ and differentiate between the major types of dementia [23, 24]. Trained neuropsychologists or research nurses administered the Korean version of the CERAD Neuropsychological Assessment Battery (CERAD-K-N) [28, 30]. The total score for the CERAD-K$\mathrm{N}$ (CERAD-TS) was obtained by summating the scores of the 6 tests: the categorical fluency test, 15-item Boston naming test, word list memory test, constructional praxis test, word list recall test, and word list recognition test [31]. We evaluated the MiniMental State Examination as a part of CERAD-K-N and calculated the difference between the Trail Making Test (TMT)-A and the TMT-B scores (TMT-D) [32, 33].

A panel of research neuropsychiatrists diagnosed dementia according to the Diagnostic and Statistical Manual of Mental Disorders, Fourth Edition (DSM-IV) diagnostic criteria [34]. They determined the subtypes of dementia according to the following criteria: AD according to the criteria of the National Institute of Neurological and Communicative Disorders and Stroke and the Alzheimer's Disease and Related Disorders Association [35], and SVD according to the DSM-IV diagnostic criteria for vascular dementia [34] and the imaging criteria proposed by Erkinjuntti et al. [36]. NC was diagnosed when the participants did not have both subjective and objective cognitive impairment. The absence of objective cognitive impairment was ascertained if a participant had a score of $>-1.5$ standard deviation for the age-, sex-, and educationadjusted norms of older adult Koreans on all neuropsychological tests.

\section{Image Acquisition and Preprocessing}

The subjects underwent 3.0 T brain MRI (Philips Medical Systems, The Netherlands). Fluid attenuation inversion recovery (FLAIR) images were obtained with the following protocol: voxel dimension $=1.0 \times 1.0 \times 3.0 \mathrm{~mm}$; repetition time $=9,900 \mathrm{~ms}$; echo time $=125 \mathrm{~ms}$; inversion time $=2,800 \mathrm{~ms}$; number of excitations $=1$; flip angle $=90^{\circ}$; field of view $=240 \mathrm{~mm}$; axial plane matrix $=$ $256 \times 256 \times 50 \mathrm{~mm}$; thickness $=3 \mathrm{~mm}$; and no interslice gap. $3 \mathrm{D}$ T1-weighted gradient-echo MR images were acquired with an echo time of $4.6 \mathrm{~ms}$, repetition time of $8.2 \mathrm{~ms}$, and a voxel dimension of $1.0 \times 1.0 \times 1.0 \mathrm{~mm}$. All data were acquired in the Digital Imaging and Communications in Medicine format and converted to ANALYZE for analysis using the import function in Statistical Parametric Mapping software version 8 (SPM8) (Wellcome Trust Center for Neuroimaging, London, http://www.fil.ion.ucl.ac.uk/ spm). 


\section{Automatic Segmentation of WMH}

The intensity inhomogeneity correction of raw FLAIR images was automatically performed using a segment routine with bias correction in SPM8. FLAIR images were resliced into $1.0 \times 1.0 \times$ $1.0 \mathrm{~mm}$ isovoxels in MATLAB 2010a (Math Works, Natick, MA, USA). WMHs on the FLAIR images were segmented using custom-written codes previously developed in-house [37] running in MATLAB and functions from SPM8, which is a fully automated segmentation procedure. Then, the FLAIR images and WMH segmentation masks of all subjects were normalized into the KNE96 template [38] using SPM8. The WMH mask was separated into several clusters using connected component labeling $[39,40]$. The total intracranial volume (TCV) was obtained using FreeSurfer (stable v6.0.0), which was used for the adjustment of the individual intracranial size variation.

\section{Shape Index}

The minimum volume for calculating the shape index per WMH cluster was $1,000 \mathrm{~mm}^{3}$. Shape feature calculations were performed for each WMH cluster as a region of interest (ROI). The volume $(V)$ was estimated as the number of voxels in the ROI times the voxel spacing;

$$
V=N \times r_{\text {lat }} \times r_{\text {cor }} \times r_{a x}
$$

Similarly, area $(A)$ was estimated as the number of boundary voxels in the ROI times the voxel spacing.

We calculated the sphericity index (SI) of the ROI's behavior in relation to a spherical object as the shape index. This was defined as follows:

$$
\mathrm{SI}=\frac{6 \sqrt{\pi} V}{A^{\frac{3}{2}}} .
$$

Thus, if the ROI's shape was similar to a sphere, the value would be close to $1(\mathrm{SI} \leq 1)$. We used the inverse of the SI (ISI) to indicate that a larger index reflected more shape irregularity and determined the ISI per subject using the mean value of the ROIs.

\section{Statistical Analysis}

The differences in characteristics among the diagnostic groups were evaluated using Pearson $\chi^{2}$ tests for categorical variables and one-way ANOVA with Bonferroni post hoc comparisons for continuous variables. We examined the associations of the volume of WMHs divided by TCV (VOL $\mathrm{WMH}_{\mathrm{WM}}$ ) and the ISI of WMHs (ISI${ }_{\mathrm{wMH}}$ ) as a dependent variable with $\mathrm{mHIS}$ as an independent variable, using univariate linear regression analyses. The significance of the differences among the regression coefficients was examined using the interaction method [41].

The associations of $\mathrm{VOL}_{\mathrm{WMH}}$ and $\mathrm{ISI}_{\mathrm{WMH}}$ with the diagnosis of $\mathrm{AD}$ and SVD were examined using multivariate multinomial regression analyses (model 1, VOL $\mathrm{WMH}_{\mathrm{W}}$ only; model 2, both VOLWMH and ISI $_{\mathrm{WMH}}$ ), with age, sex, and education as covariates. Subsequently, we compared the fitting of both models using the likelihood ratio test.

We examined the associations of $\mathrm{VOL}_{\mathrm{WMH}}$ or $\mathrm{ISI}_{\mathrm{WMH}}$ with cognitive performance within diagnostic groups using hierarchical linear regression analyses, including each cognitive test score as a dependent variable. In each hierarchical linear regression analysis, the first model included the demographic variables selected by the stepwise variable selection method among age, sex, and education to identify significant covariates, and the second (final) model add- ed $\mathrm{VOL}_{\mathrm{WMH}}$ or $\mathrm{ISI}_{\mathrm{WMH}}$ to the first model. When both $\mathrm{VOL}_{\mathrm{WMH}}$ and ISI $_{\mathrm{WMH}}$ showed significant associations with the cognitive test scores, we performed an additional hierarchical linear regression analysis. The first model included the demographic variables selected by the stepwise variable selection method, and the second model added VOL ${ }_{W M H}$ to the first. The third (final) model added ISI $_{\mathrm{WMH}}$ to the second, and we evaluated the improvement of the model fit by using the F-test.

For all analyses, VOL $\mathrm{WMH}_{\mathrm{WM}}$ and $\mathrm{ISI}_{\mathrm{WMH}}$ were natural-log transformed. A 2 -sided $p$ value of $<0.05$ was considered statistically significant. All statistical analyses were performed using $\mathrm{R}$ version 3.6.1.

\section{Results}

The ages of the participants ranged from 56 to 93 years. The Mini-Mental State Examination score was the highest in the NC group, followed by the SVD group, while mHIS was the highest in the SVD group, followed by the NC group (Table 1). The WMH total volume and VOLWMH (\% of total WMH volume from TCV) were highest in the SVD group, followed by the AD group. ISI $_{\mathrm{WMH}}$ was also the highest in the SVD group. However, in contrast to the WMH total volume and $\mathrm{VOL}_{\mathrm{WMH}}$, $\mathrm{ISI}_{\mathrm{WMH}}$ was comparable between the $\mathrm{AD}$ and $\mathrm{NC}$ groups.

The mHIS was positively associated with both VOLWMH $(\beta=0.299, \mathrm{SE}=0.061, p<0.001)$ and $\operatorname{ISI}_{\mathrm{WMH}}(\beta=$ $0.326, \mathrm{SE}=0.061, p<0.001)$. However, the standardized regression coefficients $(\beta \mathrm{s})$ were not significantly different $(p=0.749)$.

Both VOL $\mathrm{WMH}_{\mathrm{W}}$ and $\mathrm{ISI}_{\mathrm{WMH}}$ were independently associated with a diagnosis of SVD, but not with AD. As shown in model 2 in Table 2 , an increase in $\mathrm{VOL}_{\mathrm{WMH}}$ or ISI $_{\mathrm{WMH}}$ of 1 standard deviation was associated with an over 2.5 times higher risk of SVD (odds ratio $=2.597,95 \%$ confidence interval $[\mathrm{CI}]=1.342-5.026, p=0.005$, VOLwMH; odds ratio $=2.685,95 \% \mathrm{CI}=1.438-5.013, p=0.002$, ISI $\left._{\mathrm{WMH}}\right)$. The diagnosis of SVD was better explained using the model that included VOL $\mathrm{WMH}_{\mathrm{WH}}$ and $\mathrm{ISI}_{\mathrm{WMH}}$ together, rather than $\operatorname{VOL}_{\mathrm{WMH}}$ alone $\left(\chi^{2}=20.768, \mathrm{df}=2, p<\right.$ 0.001 ).

In the SVD group, both high VOL $\mathrm{WMH}_{\mathrm{WM}}$ and $\mathrm{ISI}_{\mathrm{WMH}}$ were associated with poor performance on TMT-D. The regression coefficient of ISI $_{\mathrm{WMH}}(\beta=0.308,95 \% \mathrm{CI}=$ $0.080-0.535, p=0.009$; adjusted $\left.R^{2}=0.085, p=0.008\right)$ was comparable with that of $\mathrm{VOL}_{\mathrm{WMH}}(\beta=0.293,95 \% \mathrm{CI}=$ $0.070-0.515, p=0.011$; adjusted $R^{2}=0.080, p=0.010$ ). When ISI $_{\mathrm{WMH}}$ was added to the model that included VOL $_{W M H}$, the associations of both VOL $_{\mathrm{WMH}}$ and ISI $\mathrm{WMH}_{\mathrm{WM}}$ with the TMT-D score became insignificant $(\beta=0.162$, $95 \% \mathrm{CI}=-0.147-0.472, p=0.299$ for $\mathrm{VOL}_{\mathrm{WMH}} ; \beta=0.192$, 
Table 1. Characteristics of the participants

\begin{tabular}{|c|c|c|c|c|c|}
\hline & \multirow{2}{*}{$\begin{array}{l}\mathrm{NC} \\
(N=82)\end{array}$} & \multirow{2}{*}{$\begin{array}{l}\mathrm{AD} \\
(N=82)\end{array}$} & \multirow{2}{*}{$\begin{array}{l}\text { SVD } \\
(N=82)\end{array}$} & \multicolumn{2}{|c|}{ Statistics* } \\
\hline & & & & $p$ value* & post hoc \\
\hline Age (years, mean $\pm \mathrm{SD}$ ) & $72.05 \pm 7.20$ & $76.18 \pm 6.83$ & $77.37 \pm 5.31$ & $<0.001$ & $\mathrm{NC}<\mathrm{AD}, \mathrm{SVD}$ \\
\hline Sex (female, \%) & 56.1 & 68.3 & 67.1 & 0.201 & \\
\hline Education (years, mean \pm SD) & $11.52 \pm 5.46$ & $8.30 \pm 5.66$ & $8.98 \pm 5.98$ & $<0.001$ & $\mathrm{NC}>\mathrm{AD}, \mathrm{SVD}$ \\
\hline MMSE (points, mean \pm SD) & $26.84 \pm 3.30$ & $16.61 \pm 5.61$ & $19.65 \pm 5.37$ & $<0.001$ & $\mathrm{NC}>\mathrm{SVD}>\mathrm{AD}$ \\
\hline mHIS (points, mean \pm SD) & $1.74 \pm 1.09$ & $1.59 \pm 0.61$ & $3.27 \pm 2.13$ & $<0.001$ & $\mathrm{NC}, \mathrm{AD}<\mathrm{SVD}$ \\
\hline WMH total volume, $\mathrm{cm}^{3}($ mean $\pm \mathrm{SD})$ & $12.23 \pm 12.80$ & $18.00 \pm 17.45$ & $47.98 \pm 31.55$ & $<0.001$ & $\mathrm{NC}, \mathrm{AD}<\mathrm{SVD}$ \\
\hline $\mathrm{VOL}_{\mathrm{WMH}}(\%$ of TCV, mean $\pm \mathrm{SD})$ & $0.79 \pm 0.81$ & $1.15 \pm 1.08$ & $3.05 \pm 1.99$ & $<0.001$ & $\mathrm{NC}, \mathrm{AD}<\mathrm{SVD}$ \\
\hline $\mathrm{ISI}_{\mathrm{WMH}}($ mean $\pm \mathrm{SD})$ & $3.30 \pm 0.83$ & $3.29 \pm 0.72$ & $4.58 \pm 0.99$ & $<0.001$ & $\mathrm{NC}, \mathrm{AD}<\mathrm{SVD}$ \\
\hline
\end{tabular}

$\mathrm{WMH}$, white matter hyperintensity; AD, Alzheimer's dementia; $\mathrm{ISI}_{\mathrm{WMH}}$, inverse sphericity index of WMHs (higher values indicate more irregular shape); mHIS, modified Hachinski Ischemic Score; MMSE, Mini-Mental State Examination; NC, normal cognition; SD, standard deviation; SVD, subcortical vascular dementia; TCV, total intracranial volume; $\mathrm{VOL}_{\mathrm{WMH}}$, volume of WMHs/TCV. ${ }^{*}$ One-way ANOVA for continuous variables and $\chi^{2}$ test for categorical variables with Bonferroni post hoc comparisons.

Table 2. Associations of the volume and shape of WMHs with AD and SVD

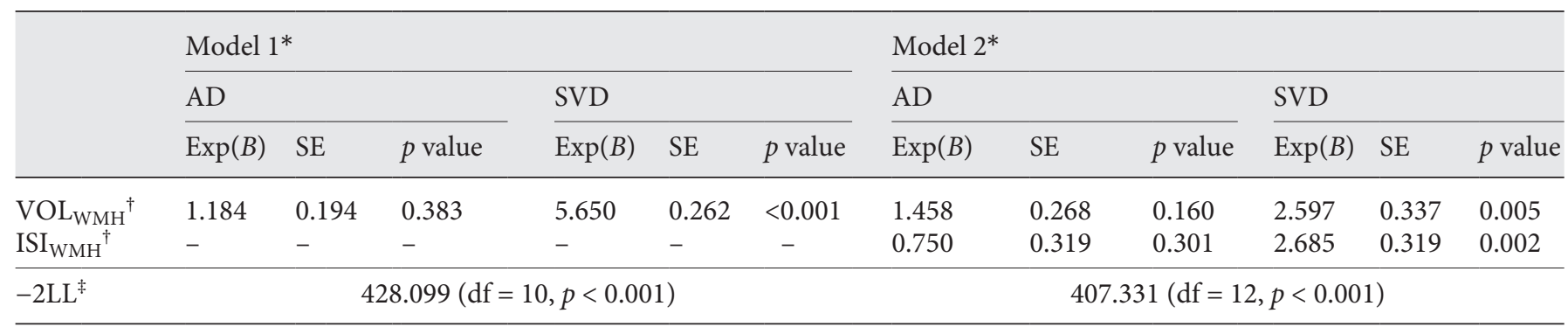

TCV, total intracranial volume; WMH, white matter hyperintensity; AD, Alzheimer's dementia; Exp (B), exponentiation of the B coefficient, an odds ratio; $\mathrm{ISI}_{\mathrm{WMH}}$, inverse sphericity index of WMHs (higher value indicates more irregular shape); SE, standard error; SVD, subcortical vascular dementia; $\mathrm{VOL}_{\mathrm{WMH}}$, volume of WMHs/TCV; -2LL: -2 log likelihood. * Multinomial regression analysis including the natural log-transformed values ${ }^{\dagger}$ of $\mathrm{VOL}_{\mathrm{WMH}}$ and $\mathrm{ISI}_{\mathrm{WMH}}$ as independent variables and age, gender, and education as covariates and employing the cognitively normal controls as the referent group. All continuous variables were $\mathrm{z}$-transformed. ${ }^{\ddagger} p$ values were derived from the likelihood ratio test by testing the null hypothesis; the likelihood ratio test between model 2 and model 1 , $\chi^{2}=$ 20.768, df $=2, p<0.001$.

$95 \% \mathrm{CI}=-0.125-0.509, p=0.232$ for ISI $_{\mathrm{WMH}}$; adjusted $\left.R^{2}=0.086, p=0.019\right)$. There was no improvement in model fit $(\mathrm{F}[1,66]=1.455, p=0.232)$. Neither $\mathrm{VOL}_{\mathrm{WMH}}$ nor ISI ${ }_{\mathrm{WMH}}$ was associated with other cognitive test scores (Table 3).

\section{Discussion}

This study demonstrated that both the volume and irregular shape of WMHs were associated with the cerebrovascular component of cognitive impairment measured by mHIS, the diagnosis of SVD, and cognitive flexibility in patients with SVD. Their associations with mHIS and cognitive performance were not significantly different. However, adding information about shape irregularity to the total volume of WMHs explained the likelihood of SVD diagnosis better.

Previous histopathological studies have demonstrated that more irregular periventricular/confluent WMHs correspond to more severe small vessel changes [11-13, $21]$, and they are associated with microcystic infarcts and patchy rarefaction of myelin, which are ischemic $[15,16]$. In contrast, periventricular caps and smooth halo are 


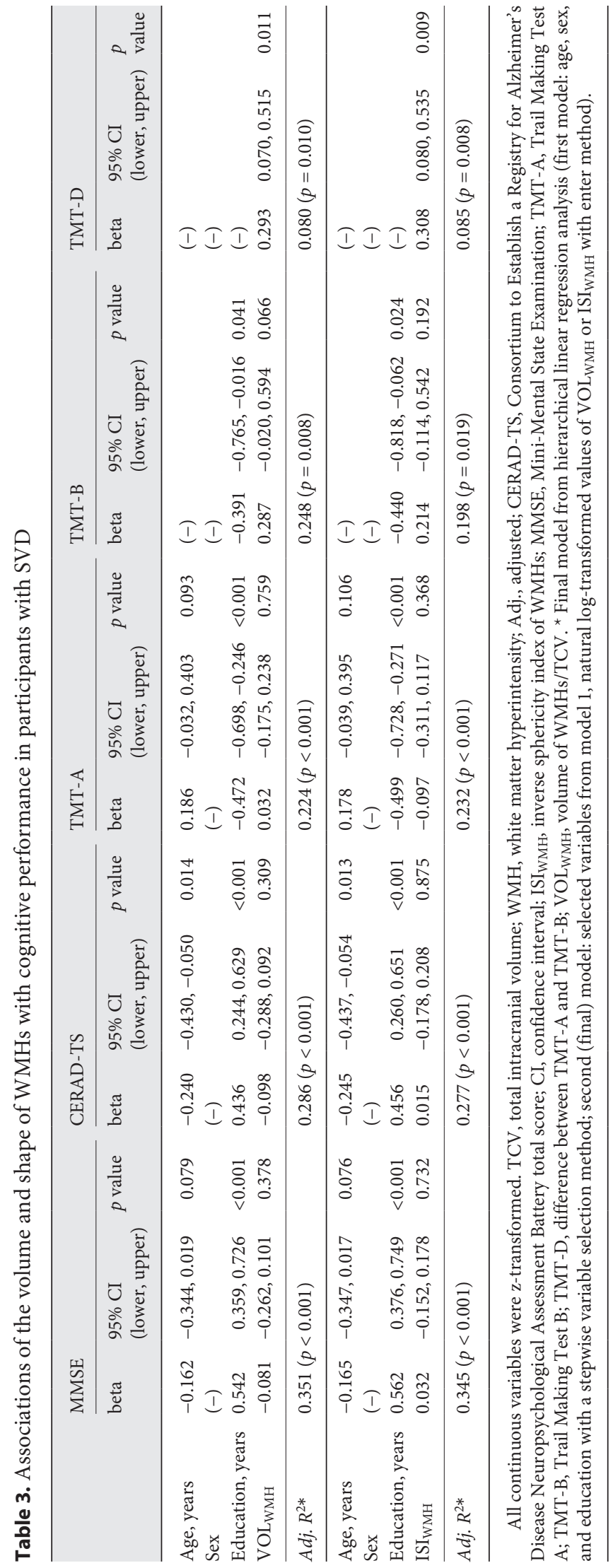

constituted of the areas of ependymal discontinuity and subependymal gliosis, which are nonischemic [12]. Punctate deep WMHs are known to represent a plethora of minimal cerebral changes that cannot be unequivocally attributed to brain ischemia [11, 14, 42-44]. Patients with type II diabetes have more non- or eccentric punctate WMH than those observed in controls, despite their $\mathrm{WMH}$ volume being comparable [20]. Patients with lacunes had more irregularly shaped confluent WMHs than those without, and this was independent of total WMH volume [21]. Together, these findings suggest that ischemic etiologies may make the shape of a WMH more irregular, which is consistent with the finding of the present study that WMHs become more irregular as the mHIS increase.

Ischemic WMHs interfere with several fasciculi connecting the posterior and anterior brain [19]. Considering the influence of the WMH penumbra, which appears normal on conventional MRI but altered on diffusion tensor imaging [45], the effect of the irregularly shaped WMHs related to ischemia on brain function may be more extensive than that of smooth or spherical WMHs. In this study, the combination of the volume and shape of WMHs facilitated a better prediction of SVD than the volume alone. Employing the quantitative measures of the volume and shape of WMH together in diagnosing SVD may narrow the gap between the presence of WMH and the clinical picture [46]. For example, people with a similar WMH volume but larger ISI may be more likely to have a high mHIS and be diagnosed with SVD. After sufficient validation using other independent populations, we may use the ISI as a supportive biomarker for vascular brain injury, such as total WMH volume, and emphasize the management of vascular risk factors in people with high ISI to prevent dementia with a high cerebrovascular component. Of course, the calculation of the shape irregularity index requires automatic segmentation of WMHs and additional MATLAB code for extracting indexes, which may limit availability and accessibility in clinical practice settings, unlike the clinical research setting.

WMH was more prevalent in $\mathrm{AD}$ patients than in cognitively normal individuals [47] and was associated with a higher risk of incident $\mathrm{AD}$ in individuals with $\mathrm{NC}$ or mild cognitive impairment [47]. WMH as a vascular pathology may act additively with amyloid pathology in progressing from the preclinical to clinical stages in $\mathrm{AD}$ and related disorders [48-50]. However, neither the volume nor shape of WMHs was associated with the diagnosis of $\mathrm{AD}$ in this study. This may be attributed to the 
limited sample size or the preceding effect of WMHs before amyloidosis or neurodegeneration across the $\mathrm{AD}$ pathophysiology cascade. AD patients showed a higher $\mathrm{WMH}$ volume than NC individuals, but their WMH shapes were comparable with those of the controls in this study, suggesting that WMHs in AD patients may be less likely to be ischemic than WMHs in SVD and may be related to other pathologies, such as Wallerian degeneration [51].

In the patients with SVD, both $\mathrm{VOL}_{\mathrm{WMH}}$ and $\mathrm{ISI}_{\mathrm{WMH}}$ were associated with TMT-D, indicating that mental flexibility may be worse when the WMH volume is higher and the shape is irregular. TMT-D, an index of the level of interference by the addition of the flexibility component of TMT-B [33], is a more accurate measure of executive skills than TMT-B $[32,52]$. Unlike the gain in model fit for SVD diagnosis observed after adding ISI $_{\mathrm{WMH}}$ to the model that included $\mathrm{VOL}_{\mathrm{WMH}}$, adding ISI $_{\mathrm{WMH}}$ alone did not show a significant improvement in model fit for predicting TMT-D score among SVD patients. The additional effect of information about WMH shape on accounting for cognitive test scores may be limited in SVD patients due to their substantial WMH volumes. Meanwhile, the insignificant association with TMT-A is different from the finding of a previous study that used the COSU as a measure of WMH shape irregularity; this may be attributed to the difference in the diagnoses of the population [19].

This study has some limitations. First, it was a crosssectional study, and the causal relationships should be confirmed in future prospective studies. Second, we did not consider the impact of other small vessel diseases (lacunes and microbleeds), neurodegeneration (hippocampal atrophy), or Alzheimer's disease biomarkers (amyloid or tau). Third, there may be various indices besides $\mathrm{ISI}_{\mathrm{WMH}}$ that represent shape irregularity. We chose ISI $_{W M H}$ because of the simplicity of directionality (i.e., the larger, more irregular) and ease of use in clinical practice. Fourth, there was a high correlation between ISI ${ }_{\mathrm{WMH}}$ and $\mathrm{VOL}_{\mathrm{WMH}}(r=0.75, p<0.001$, by Pearson's correlation) in our population, which may have contributed to the loss of significant associations between both variables and the TMT-D score, when both variables were included simultaneously as predictors for the same model. Further studies using the population stratified by the $\mathrm{WMH}$ volume are needed to validate the present findings. Fifth, we administered only the TMT to evaluate the executive function, which is not enough to evaluate diverse aspects of executive function. Sixth, the analyses were not adjusted for multiple comparisons, which may have in- creased the likelihood of a type I error. Finally, the underlying etiology for the association between the WMH shape and cognitive impairment was not investigated directly.

\section{Conclusion}

The irregularity of the shape of WMHs may correlate with the high cerebrovascular component of cognitive impairment and the diagnosis and low cognitive flexibility of SVD. The WMH shape may improve the prediction of SVD diagnosis when used in combination with the WMH volume.

\section{Statement of Ethics}

All subjects were fully informed of the study protocol and provided written informed consent to participate, signed by either the participant or their legal guardians. This study's protocol was approved by the Institutional Review Board of Seoul National University Bundang Hospital (IRB No. B-1505/297-001).

\section{Conflict of Interest Statement}

The authors have no conflicts of interest to declare.

\section{Funding Sources}

This research was supported by Grants No. 13-2015-022 from the SNUBH Research Fund, No. 2015R1D1A1A0105925 from the Basic Science Research Program through the National Research Foundation of Korea funded by the Ministry of Education, and no. HI09C1379 from the Korean Health Technology R\&D Project, Ministry of Health and Welfare, Republic of Korea.

\section{Author Contributions}

J.W.H., H.L., and K.W.K. contributed to the study concept and design, analysis and interpretation of data, and manuscript elaboration. H.K., S.L., and H.L. contributed to the imaging analysis and original draft preparation. J.W.H., G.E.K., J.B.B., S.W.S., K.W.K., and J.H.K. contributed to the acquisition and analysis of clinical and imaging data. 


\section{References}

1 Rostanski SK, Zimmerman ME, Schupf N, Manly JJ, Westwood AJ, Brickman AM, et al. Sleep disordered breathing and white matter hyperintensities in community-dwelling elders. Sleep. 2016 Apr;39(4):785-91.

2 Sabri O, Ringelstein EB, Hellwig D, Schneider R, Schreckenberger M, Kaiser HJ, et al. Neuropsychological impairment correlates with hypoperfusion and hypometabolism but not with severity of white matter lesions on MRI in patients with cerebral microangiopathy. Stroke. 1999 Mar;30(3):556-66.

3 Garde E, Mortensen EL, Krabbe K, Rostrup E, Larsson $\mathrm{HB}$. Relation between age-related decline in intelligence and cerebral white-matter hyperintensities in healthy octogenarians: a longitudinal study. Lancet. 2000 Aug; 356(9230):628-34.

4 Schmidt R, Schmidt H, Kapeller P, Lechner A, Fazekas F. Evolution of white matter lesions. Cerebrovasc Dis. 2002;13 Suppl 2(Suppl 2): 16-20.

5 Tullberg M, Fletcher E, DeCarli C, Mungas D, Reed BR, Harvey DJ, et al. White matter lesions impair frontal lobe function regardless of their location. Neurology. 2004 Jul;63(2): 246-53.

6 Biesbroek JM, Weaver NA, Hilal S, Kuijf HJ, Ikram MK, Xu X, et al. impact of strategically located white matter hyperintensities on cognition in memory clinic patients with small vessel disease. PLoS One. 2016 Nov;11(11):e0166261.

7 Lampe L, Kharabian-Masouleh S, Kynast J, Arelin K, Steele CJ, Löffler M, et al. Lesion location matters: the relationships between white matter hyperintensities on cognition in the healthy elderly. J Cereb Blood Flow Metab. 2019 Jan;39(1):36-43.

8 Jiang J, Paradise M, Liu T, Armstrong NJ, Zhu W, Kochan NA, et al. The association of regional white matter lesions with cognition in a community-based cohort of older individuals. Neuro Image. 2018 Mar 29;19:14-21.

9 Biesbroek JM, Weaver NA, Biessels GJ. Lesion location and cognitive impact of cerebral small vessel disease. Clin Sci. 2017 Apr 25; 131(8):715-28.

10 Biesbroek JM, Lam BYK, Zhao L, Tang Y, Wang Z, Abrigo J, et al. High white matter hyperintensity burden in strategic white matter tracts relates to worse global cognitive performance in community-dwelling individuals. J Neurol Sci. 2020 Jul 15;414:116835.

11 Fazekas F, Kleinert R, Offenbacher $H$, Schmidt R, Kleinert G, Payer F, et al. Pathologic correlates of incidental MRI white matter signal hyperintensities. Neurology. 1993 Sep;43(9):1683-9.

12 Kim KW, MacFall JR, Payne ME. Classification of white matter lesions on magnetic resonance imaging in elderly persons. Biol Psychiatry. 2008 Aug;64(4):273-80.

13 Gouw AA, Seewann A, van der Flier WM, Barkhof F, Rozemuller AM, Scheltens P, et al. Heterogeneity of small vessel disease: a sys- tematic review of MRI and histopathology correlations. J Neurol Neurosurg Psychiatry. 2011 Feb;82(2):126-35.

14 Fazekas F, Kleinert R, Offenbacher H, Payer F, Schmidt R, Kleinert G, et al. The morphologic correlate of incidental punctate white matter hyperintensities on MR images. AJNR Am J Neuroradiol. 1991 Sep-Oct;12(5):91521.

15 van Swieten JC, van den Hout JH, van Ketel BA, Hijdra A, Wokke JH, van Gijn J. Periventricular lesions in the white matter on magnetic resonance imaging in the elderly. A morphometric correlation with arteriolosclerosis and dilated perivascular spaces. Brain. 1991 Apr;114 (Pt 2)(Pt 2):761-74.

16 Thomas AJ, O'Brien JT, Barber R, McMeekin W, Perry R. A neuropathological study of periventricular white matter hyperintensities in major depression. J Affect Disord. 2003 Sep;76(1-3):49-54.

17 Schmidt R, Schmidt H, Haybaeck J, Loitfelder M, Weis S, Cavalieri M, et al. Heterogeneity in age-related white matter changes. Acta Neuropathol. 2011 Aug;122(2):171-85.

18 Pantoni L. Cerebral small vessel disease: from pathogenesis and clinical characteristics to therapeutic challenges. Lancet Neurol. 2010 Jul;9(7):689-701.

19 Lange C, Suppa P, Mäurer A, Ritter K, Pietrzyk $\mathrm{U}$, Steinhagen-Thiessen E, et al. Mental speed is associated with the shape irregularity of white matter MRI hyperintensity load. Brain Imaging Behav. 2017 Dec;11(6):1720-30.

20 de Bresser J, Kuijf HJ, Zaanen K, Viergever MA, Hendrikse J, Biessels GJ, et al. White matter hyperintensity shape and location feature analysis on brain MRI; proof of principle study in patients with diabetes. Sci Rep. 2018 Jan;8(1):1893.

21 Ghaznawi R, Geerlings MI, Jaarsma-Coes MG, Zwartbol MH, Kuijf HJ, van der Graaf $\mathrm{Y}$, et al. The association between lacunes and white matter hyperintensity features on MRI: the SMART-MR study. J Cereb Blood Flow Metab. 2019 Dec;39(12):2486-2496.

22 Gwo CY, Zhu DC, Zhang R. Brain white matter hyperintensity lesion characterization in T2 fluid-attenuated inversion recovery magnetic resonance images: shape, texture, and potential growth. Front Neurosci. 2019;13: 353.

23 Rosen WG, Terry RD, Fuld PA, Katzman R, Peck A. Pathological verification of ischemic score in differentiation of dementias. Ann Neurol. 1980 May;7(5):486-8.

24 Goodman A. Vladimir Hachinski: a pioneer in the field of stroke. Lancet Neurol. 2009 Jan; 8(1):34.

25 Hachinski V, Oveisgharan S, Romney AK, Shankle WR. Optimizing the Hachinski ischemic scale. Arch Neurol. 2012 Feb;69(2):16975.

26 Kim YH, Kwon OD. Clinical correlates of Hachinski ischemic score and vascular factors in cognitive function of elderly. Biomed Res Int. 2014;2014:852784.

27 Han JW, Kim TH, Kwak KP, Kim K, Kim BJ, Kim SG, et al. Overview of the Korean longitudinal study on cognitive aging and dementia. Psychiatry Investig. 2018 Aug;15(8):76774.

28 Lee JH, Lee KU, Lee DY, Kim KW, Jhoo JH, $\mathrm{Kim} \mathrm{JH}$, et al. Development of the Korean version of the consortium to establish a registry for Alzheimer's disease assessment packet (CERAD-K): clinical and neuropsychological assessment batteries. J Gerontol B Psychol Sci Soc Sci. 2002 Jan;57(1):P47-53.

29 Takahashi M, Oda Y, Sato K, Shirayama Y. Vascular risk factors and the relationships between cognitive impairment and hypoperfusion in late-onset Alzheimer's disease. Acta Neuropsychiatr. 2018 Dec;30(6):350-8.

30 Lee DY, Lee KU, Lee JH, Kim KW, Jhoo JH, Kim SY, et al. A normative study of the CERAD neuropsychological assessment battery in the Korean elderly. J Int Neuropsychol Soc. 2004 Jan;10(1):72-81.

31 Han JY, Seo EH, Yi D, Sohn BK, Choe YM, Byun MS, et al. A normative study of total scores of the CERAD neuropsychological assessment battery in an educationally diverse elderly population. Int Psychogeriatr. 2014; 26(11):1897-904

32 Ble A, Volpato S, Zuliani G, Guralnik JM, Bandinelli S, Lauretani F, et al. Executive function correlates with walking speed in older persons: the InCHIANTI study. J Am Geriatr Soc. 2005 Mar;53(3):410-5.

33 Bowie CR, Harvey PD. Administration and interpretation of the trail making test. Nat Protoc. 2006;1(5):2277-81.

34 American Psychiatric Association. Diagnostic and statistical manual of mental disorders. 4th ed. Washington, DC: American Psychiatric Publishing, Inc.; 1994.

35 McKhann G, Drachman D, Folstein M, Katzman R, Price D, Stadlan EM. Clinical diagnosis of Alzheimer's disease: report of the NINCDS-ADRDA work group under the auspices of department of health and human services task force on Alzheimer's disease. Neurology. 1984;34(7):939-44.

36 Erkinjuntti T, Inzitari D, Pantoni L, Wallin A, Scheltens P, Rockwood K, et al. Research criteria for subcortical vascular dementia in clinical trials. J Neural Transm Suppl. 2000;59: 23-30.

37 Yoo BI, Lee JJ, Han JW, Oh SY, Lee EY, MacFall JR, et al. Application of variable threshold intensity to segmentation for white matter hyperintensities in fluid attenuated inversion recovery magnetic resonance images. Neuroradiology. 2014;56(4):265-81.

38 Lee H, Yoo BI, Han JW, Lee JJ, Oh SY, Lee EY, et al. Construction and validation of brain MRI templates from a Korean normal elderly population. Psychiatry Investig. 2016;13(1): 135-45. 
39 Haralick RM, Shapiro LG. Computer and robot vision: Addison-Wesley Longman Publishing Co., Inc.; 1992. p. 28-48.

40 Suzuki K, Horiba I, Sugie N. Linear-time connected-component labeling based on sequential local operations. Comput Vis Image Underst. 2003 Jan;89(1):1-23.

41 Clifford C, Clogg EP, Haritou A. Statistical methods for comparing regression coefficients between models. Am J Sociol. 1995; 100(5):1261-93.

42 Awad IA, Johnson PC, Spetzler RF, Hodak JA. Incidental subcortical lesions identified on magnetic resonance imaging in the elderly. II. Postmortem pathological correlations. Stroke. 1986 Nov-Dec;17(6):1090-7.

43 Veldink JH, Scheltens P, Jonker C, Launer LJ. Progression of cerebral white matter hyperintensities on MRI is related to diastolic blood pressure. Neurology. 1998 Jul;51(1):319-20.

44 Schmidt R, Fazekas F, Kapeller P, Schmidt H, Hartung HP. MRI white matter hyperintensities: three-year follow-up of the Austrian stroke prevention study. Neurology. 1999 Jul; 53(1):132-9.
45 Maniega SM, Valdés Hernández MC, Clayden JD, Royle NA, Murray C, Morris Z, et al. White matter hyperintensities and normalappearing white matter integrity in the aging brain. Neurobiol Aging. 2015 Feb;36(2):90918.

46 Prins ND, Scheltens P. White matter hyperintensities, cognitive impairment and dementia: an update. Nat Rev Neurol. 2015 Mar; 11(3):157-65

47 Wang YL, Chen W, Cai WJ, Hu H, Xu W, Wang ZT, et al. Associations of white matter hyperintensities with cognitive decline: a longitudinal study. J Alzheimers Dis. 2020;73(2): 759-68.

48 Provenzano FA, Muraskin J, Tosto G, Narkhede A, Wasserman BT, Griffith EY, et al. White matter hyperintensities and cerebral amyloidosis: necessary and sufficient for clinical expression of Alzheimer disease? JAMA Neurol. 2013 Apr;70(4):455-61.
49 Gordon BA, Najmi S, Hsu P, Roe CM, Morris JC, Benzinger TL. The effects of white matter hyperintensities and amyloid deposition on Alzheimer dementia. Neuroimage Clin. 2015; 8:246-52.

50 Soldan A, Pettigrew C, Zhu Y, Wang MC, Moghekar A, Gottesman RF, et al. White matter hyperintensities and CSF Alzheimer disease biomarkers in preclinical Alzheimer disease. Neurology. 2020 Mar; 94(9):e950-60.

51 McAleese KE, Walker L, Graham S, Moya ELJ, Johnson M, Erskine D, et al. Parietal white matter lesions in Alzheimer's disease are associated with cortical neurodegenerative pathology, but not with small vessel disease. Acta Neuropathol. 2017 Sep;134(3): 459-73.

52 Drane DL, Yuspeh RL, Huthwaite JS, Klingler LK. Demographic characteristics and normative observations for derived-trail making test indices. Neuropsychiatry Neuropsychol Behav Neurol. 2002 Mar;15(1):39-43. 